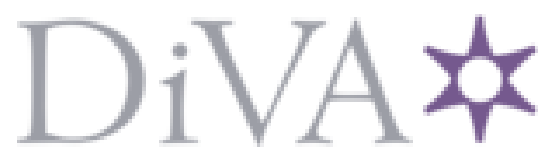

http://www.diva-portal.org

This is the published version of a paper presented at IEEE PES GM 2014.

Citation for the original published paper:

Almas, M., Vanfretti, L. (2014)

Open Source SCADA Implementation and PMU Integration for Power System Monitoring and Control Applications.

In:

N.B. When citing this work, cite the original published paper.

Permanent link to this version:

http://urn.kb.se/resolve?urn=urn:nbn:se:kth:diva- 157123 


\title{
Open Source SCADA Implementation and PMU Integration for Power System Monitoring and Control Applications
}

\author{
M.S. Almas, L. Vanfretti, Stig Løvlund, and J.O. Gjerde
}

\begin{abstract}
This paper presents the implementation of an Open Source SCADA system in a laboratory and discusses methods for PMU data integration into SCADA. SCADA BR is a webbrowser based SCADA that enables the user to access monitoring, control and automation equipment over multiple protocols. For implementation, protection relays from SEL are configured as DNP3 outstations to act as slaves and SCADA BR which is installed in one of the workstations in the laboratory acts as master. The evaluation of SCADA BR has been performed by executing a power system model in a real-time simulator and coupling its analog outputs with the protection relays. The integration of PMU measurements in the SCADA system and their use for monitoring is discussed. The limitations of the SCADA systems to fully utilize PMU data are also presented.
\end{abstract}

Index Terms-SCADA, Distributed Network Protocol, RealTime Simulation, Hardware-in-the-Loop Simulation, Open Source, Synchronized Phasor Measurements, PMU

\section{INTRODUCTION}

Supervisory Control and Data Acquisition (SCADA) provides real-time data exchange between a control/monitoring center (master) and field devices (slaves) [1]. In this Master-Slave architecture, the master, which is a machine where the SCADA software is configured, polls the field devices at regular intervals and updates the operator with the latest status provided by the field devices. This gives a holistic overview of the state of the system to the operator. In addition SCADA allows the operator to take control actions by sending commands to field devices. The master logs and records all events in a file and generates automatic reports and notifications in case of critical situations. It also provides visual and audio alarms in case a certain measurement from a field device has violated the threshold limits set by the operator. Because of the familiarity of operators with SCADA systems, and the availability of PMU measurements, it appears attractive to incorporate PMU data into the SCADA system to support traditional power system monitoring and control applications.

Most of the modern protection relays support several protocols, e.g. Modbus and DNP 3.0 [2] to serve as data sources for SCADA master. These protection relays can be configured to send a data set involving analog quantities and digital status to the SCADA master when polled. Commercially available systems are costly and difficult to use

This work was supported in part by Nordic Energy Research through the STRONg ${ }^{2}$ rid project and by Statnett SF, the Norwegian TSO.

M. S. Almas, and L. Vanfretti are with KTH Royal Institute of Technology, Stockholm, Sweden. (e-mail: \{msalmas, luigiv\}@kth.se)

L. Vanfretti, S. Løvlund and J.O. Gjerde are with Statnett SF, Research and Development, Oslo, Norway (email: \{luigi.vanfretti, stig.lovlund, jan.gjerde\}@statnett.no for research purposes in the laboratory. Thus, an open source SCADA implementation for development of monitoring and control application in the laboratory appears attractive. This paper describes the implementation of open source SCADA in the Smart Transmission Systems Lab (SmarTS-Lab) at KTH, Sweden. Open source SCADA is coupled with protection relays from SEL and its performance is evaluated using realtime hardware-in-the-loop simulation (RT-HIL).

The paper is organized as follows: Section II provides information about the integration of an open source SCADA system in SmarTS-Lab. Section III presents the configuration of SCADA BR and modifications required in protection relay settings to use them as DNP3 sources. The performance of SCADA BR is evaluated using RT-HIL simulation for a twoarea four machine power system in Section IV. Section V discusses the integration of PMU data and applications with the SCADA system. Finally in Section VI, conclusions are drawn and future work is summarized.

\section{INTEGRATION OF OPEN SOURCE SCADA IN SMARTS-LAB}

This section provides an update on the integration of open source SCADA BR [9] in Smart Transmission System Laboratory (SmarTS-Lab) established at KTH as reported in [3]. This laboratory is equipped with protection relays/PMUs from SEL, ABB and Arbiter. All these devices are DNP3 compliant and can be configured as DNP3 outstations to stream out their analog and digital status. All these PMUs can be coupled with Opal-RT's eMEGAsim real-time simulator to acquire synchrophasor measurements for a simulated scenario.

SCADA BR is configured in SmarTS-Lab on one of the workstations in the lab to acquire the status updates from these PMUs/relays using DNP3 for monitoring and control purposes. The architecture is shown in Figure 1 with SCADA master polling all the PMUs at a specific polling rate to acquire updates for analog quantities and digital status.

\section{OPEN SOURCE SCADA BR}

In order to configure SCADA BR to communicate with protection relays for status update, several steps need to be performed as discussed below.

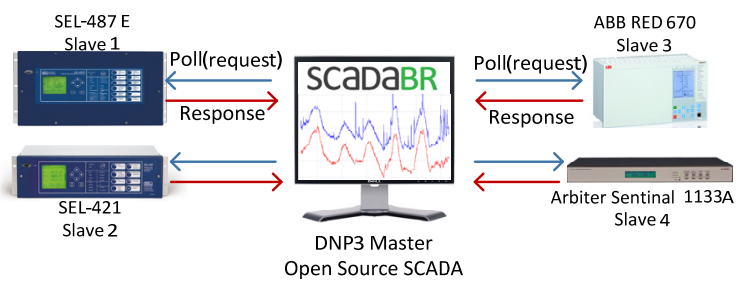

Fig.1: Architectural integration of Open Source SCADA in SmarTS-Lab 


\section{A. Configuring Protection Relays as DNP3 Outstations}

Outstations act as Slaves and reply to the Master when polled. SmarTS-Lab is equipped with three protection relays from SEL [10] which are DNP3 compliant and provide Level 2 Outstation interface for direct network connections to the relay. DNP3 settings are made by using SEL's AcSELerator Quickset software [11]. The configuration for the SEL-421 protection relay is shown in Figure 2. These settings enable the DNP3 feature on a particular port of the relay, providing a DNP or Slave address to it, and reserving a port for data transmission using either TCP or UDP. SEL relays allow the user to either use the default DNP3 mappings, or to remap the DNP3 data from a default map and organize it into a smaller data set as optimized for user applications. Commonly configured data sets include analog quantities (voltage, current, real power and frequency measurements) and digital quantities (status of different protection functions of protection relays). These mappings can be readily changed according to the experimental setup and requirements.

\section{B. Adding DNP3 Outstations in Open Source SCADA}

Once the protection relays are configured and appropriate DNP3 data set is selected, these DNP3 outstations are added to the SCADA as DNP3 sources. For this study, SCADA BR is installed and configured in a workstation in SmarTS-Lab. This workstation acts as a Master and polls all Slaves at regular interval (or polling time) to acquire information and present it to the user. Figure 3 shows important configurations to add protection relays as DNP3 data sources to SCADA BR which are similar to those of the protection relays.

Once the protection relay is added to SCADA BR, data points are added to each DNP3 source. These data points are those configured in the protection relays during DNP3 mapping. SCADA BR differentiates multiple data points from the same source depending upon their index number. The index number represents the register that the data point holds in the current mapping of protection relay. Figure 3 shows the data points configured for one of the SEL-421 relays for this study.

\section{Alarms, Graphics and Event Handler}

SCADA BR allows adding alarms on each data point so that the user can obtain both visual and audio notification

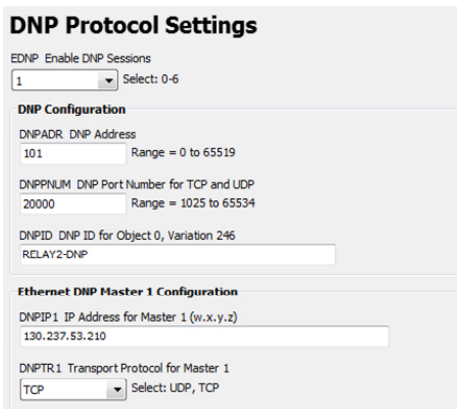

Fig.2: SEL-421 settings for enabling DNP3 features when a certain measurement exceeds its limit. In addition SCADA BR provides the functionality to start logging the data as soon as the alarm is raised. This is similar to event recording in protection relays. By using different threshold levels for a particular measurement, the alarms can be configured to provide urgent, critical or life safety alarms. With SCADA BR, the user can upload a single line diagram of his power system for graphical representation of selected data points of interest.

\section{Testing Open Source SCADA}

The performance and features of SCADA BR are evaluated using RT-HIL simulation of a two area four machine power system. Figure 5 shows the single line diagram of the test case. Areal and Area 2 consist of two generators and local loads each. However the load in Area 2 is larger than the local generation, so there is a power transfer from Area 1 to Area 2. In addition a part of load at bus 2 is randomly varied to expect changes in load current which will be captured by SCADA BR using DNP3.

The three phase voltage and currents from Bus 1 and Bus 2 were sent to the VT and CT inputs of two different SEL-421 protection relays to monitor the two DNP3 sources, which communicate with a common Master for status updates. The low level voltages and currents outputs from analog output of Opal-RT's eMEGAsim ( $\pm 16 \mathrm{~V}$ and $\pm 20 \mathrm{~mA})$ are amplified by using current and voltage amplifiers from Megger to standard secondary injections $( \pm 100 \mathrm{~V}$ and $\pm 1 \mathrm{~A})$ to the VT and CT input of the protection relays. The workstation where SCADA BR is configured polls for status updates at every 1 sec. At each poll, the protection relays reply with analog quantities and the status of protection functions. Figure 4 shows the experimental setup and data flow for RT-HIL simulation.

Alarms were configured for this study using high threshold for analog quantities. SCADA BR should provide a flag (critical alarm) when the analog quantities exceed their threshold. For digital status, a critical alarm was configured for a status change from ' 0 ' to ' 1 ' (i.e. when the relay protection function has operated). Finally for graphical visualization, the single line diagram along with LEDs and data points' measurements was configured in SCADA BR. Figure 5 shows the graphical interface developed for this study.

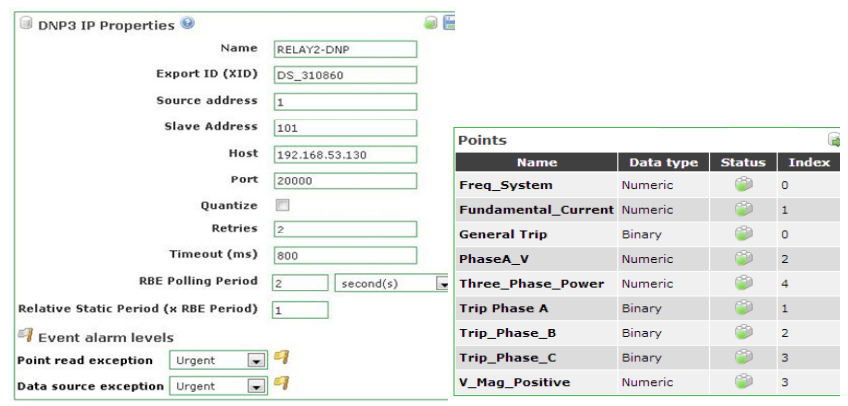

Fig.3: Adding protection relays to SCADA BR as DNP3 data sources and 
configuring analog and digital data points associated with SEL-421.

The yellow bulb in Area 1 represents the voltage which in steady state shows $230 \mathrm{kV}$. The first LED on the transmission line shows the current flowing through this line (1498 A). The second LED on transmission line represents the frequency of power system $(50 \mathrm{~Hz})$. The fan represents the power flow from Area 1 to Area 2 (1034 MW). The bulb and the two yellow LEDs are activated if any of these measurements exceed their threshold. The four LEDs in series represent General Trip, Phase A Trip, Phase B Trip and Phase $\mathrm{C}$ Trip of the transmission line respectively. These are digital status currently showing ' 0 ' i.e., none of the protection function has operated.

To further evaluate the performance of SCADA BR, a three phase to ground fault was introduced near Bus 1 in the model executing in RT-HIL. This resulted in trip of the protection relay (on the right hand side) for instantaneous overcurrent protection of all phases. This terminated the simulation by opening the breaker at both ends of the transmission line in the model. SCADA BR logs this instability in real-time and allows the user to view it in graphical form. Figure 6 shows the graphical representation of analog and digital data points within a 2 minute window of the simulation. The plots show that at 20:04:15, the three phase power and voltage magnitude dropped to zero and at the same time all the digital status changed from ' 0 ' to ' 1 ', i.e. the protection relay has operated for over current protection for all the three phases as shown in Figure 6. At the same time, the watch-list in Figure 7 shows the alarms that rose due to the violation of the configured thresholds, and the time of the status change. In addition the alarm generates a beep sound every 1 second after activation to gain user attention. None of the alarms rose when the current exceeded its higher threshold because the polling rate for the Master in the study was set to 1 second while the tripping time of SEL-421 for over current protection is between 23 26 msec. DNP3 could not capture the increase in current at Bus 1 due to a three phase to ground fault.

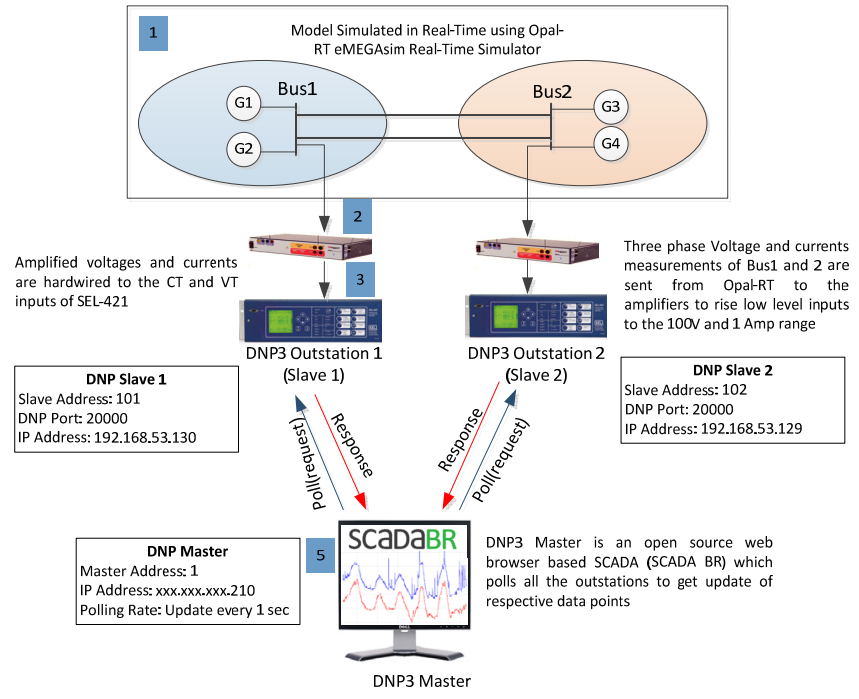

Fig.4: Data workflow for the test case simulation

\section{INTEGRATION OF PMU DATA INTO SCADA SYSTEM}

It is clear from Section IV, that by using traditional polling rates of 1 3 seconds for SCADA update, very little information can be gathered about the analog quantities in case of transients because the protection relay tripping time is much faster $(20 \sim 40 \mathrm{msec})$. In order to extract maximum information from the protection relays, synchrophasor measurements were added into the SCADA system. Firstly the PMU data set of SEL-421 was configured to add analog quantities and digital signals at a reporting rate of 50 samples per second.

These PMU measurements were received in workstation in LabView environment using Statnett's Synchrophasor Software Development Kit $\mathrm{S}^{3} \mathrm{DK}$ [12] which provides realtime synchrophasor data in the LabView environment and provides access to raw measurements of all the analog, digital and phasor quantities available in PMU/PDC streams. Within the LabView environment, these raw measurements from synchrophasors were written in a text file which keeps updating all the entries after each $20 \mathrm{msec}$. These text files were read in SCADA BR with an update period of $20 \mathrm{msec}$. The overall process is shown in Figure 8. SCADA data points were updated to include the synchrophasor measurements from the PMUs and the same alarm limits were imposed on this new data as for the DNP3 measurements. SCADA BR measurements were also published to remote HMI / SCADA clients (another workstation in the lab) and the iPhone for remote monitoring of SCADA updates. This was carried out by publishing SCADA data points to Pachube [13] which is a simple and secure RESTful API for sharing sensor's and actuator's data over internet. This data stream was received at remote ends using Pachube Data Dashboard Application [14]. A Similar scenario was executed again and this time, the PMU based measurements showed the trend of current during the fault duration while DNP3 based analog measurements didn't raise any alarm. The waveforms are shown in Figure 9. It clearly shows that with integration of synchrophasor measurements into SCADA, the power system operators can have improvements in their traditional monitoring applications.

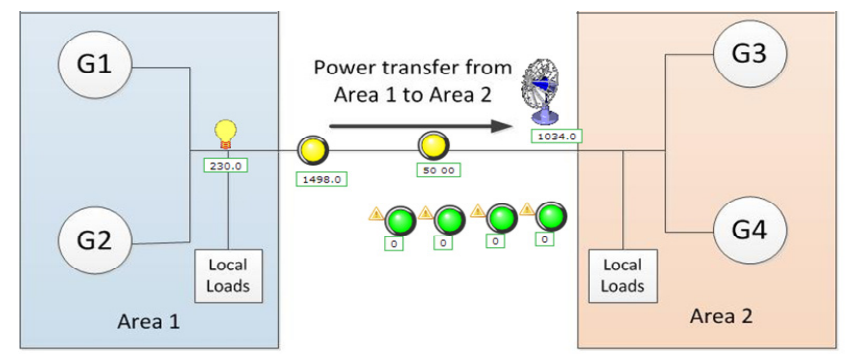

Fig.5: Graphical view of test case developed in SCADA BR showing analog quantities and protection function status in real-time 

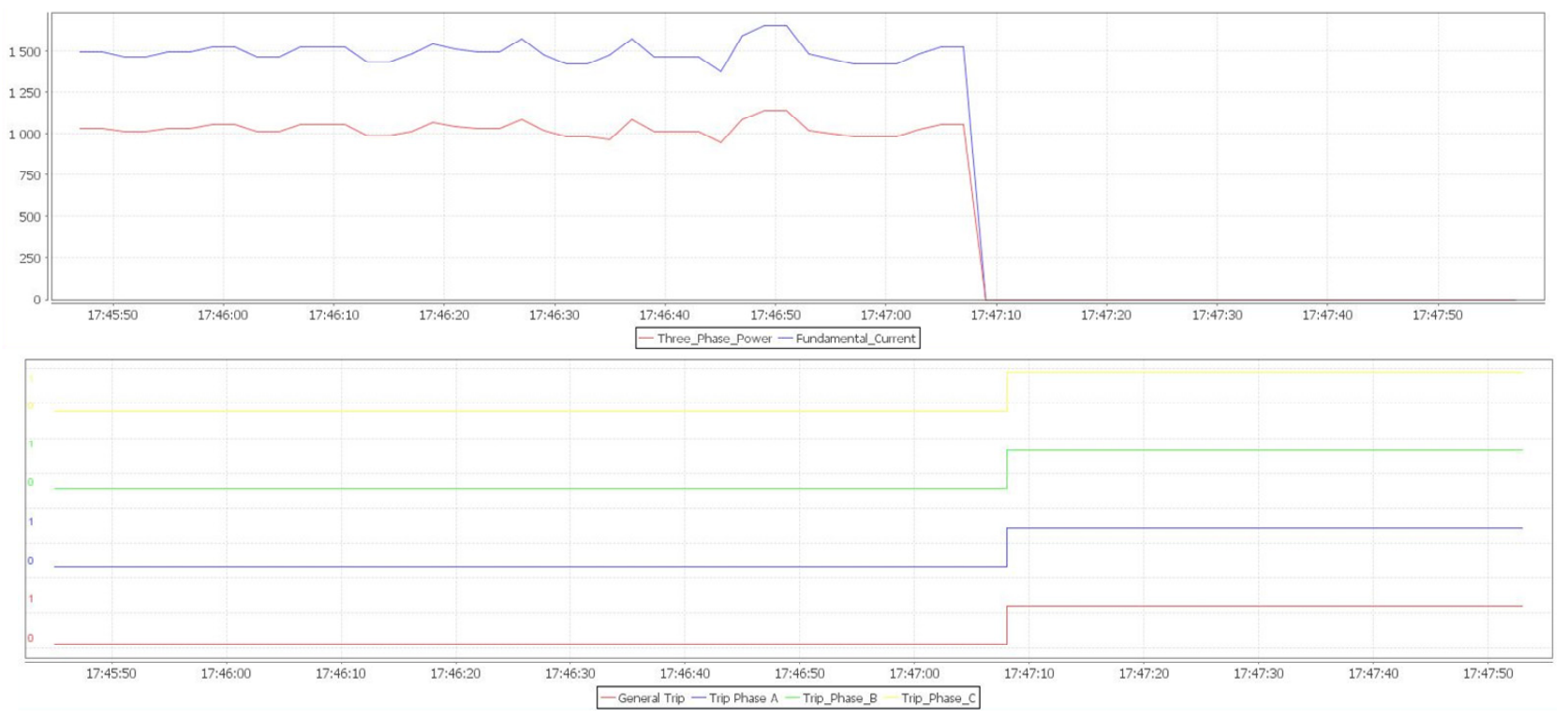

Fig.6: Plots showing the two minute window captured by SCADA BR during the RT-HIL simulation. The top plot shows the trend of the three phase real power (in $\mathrm{MW}$ ) and voltage (in $\mathrm{kV}$ ). The bottom plot shows the status of digital signals representing operation of protection functions i.e. overcurrent protection for all the three phases at 20:04:15

\begin{tabular}{|c|c|c|c|}
\hline RELAY2-DNP - General Trip & 1 & $17: 47: 53$ & $\nabla \otimes \Delta \circ$ \\
\hline \multicolumn{4}{|l|}{ 17:47:10 - General Trip has been 1 for more than 1 second(s) } \\
\hline RELAYZ-DNP - Trip Phase A & 1 & $17: 47: 53$ & D $8 \nabla 0$ \\
\hline \multicolumn{4}{|l|}{ 17:47:10 - Trip Phase A has been 1 for more than 1 second(s) } \\
\hline RELAY2-DNP - Trip_Phase_B & 1 & $17: 47: 53$ & 目 $8 \Delta \circ$ \\
\hline \multicolumn{4}{|l|}{ 17:47:10 - Trip_Phase_B has been 1 for more than 1 second(s) } \\
\hline RELAY2-DNP - Trip_Phase_C & 1 & $17: 47: 53$ & 四 $8 \Delta \circ$ \\
\hline \multicolumn{4}{|l|}{ 17:47:10 - Trip_Phase_C has been 1 for more than 1 second(s) } \\
\hline RELAY2-DNP - Freq_System & 4998 & $17: 47: 53$ & $\nabla \oplus \Delta \circ$ \\
\hline
\end{tabular}

Fig.7: Watch-list showing the alarms that rose for all digital status at 20:04:15. The orange flags show that these are critical events. The user can either acknowledge these alarms or silence them.

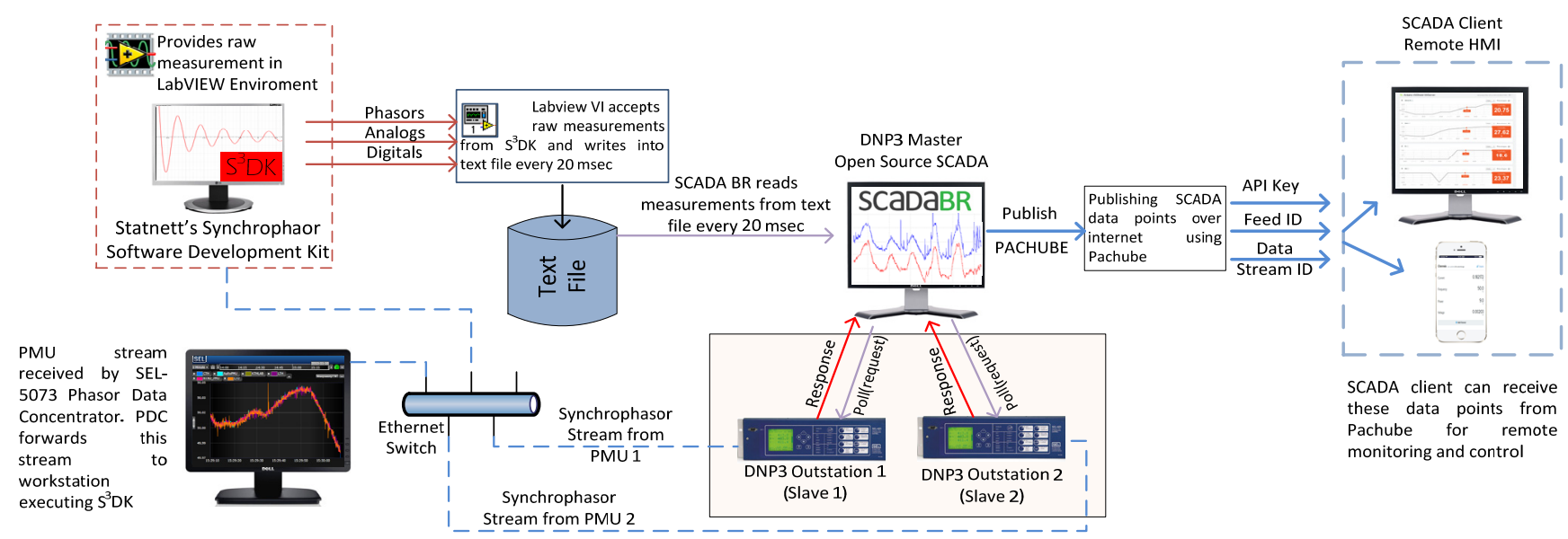

Fig.8: Integrating PMU data into SCADA BR using $\mathrm{S}^{3} \mathrm{DK}$ and publishing SCADA to remote HMI/SCADA Client and iPhone using Pachube 


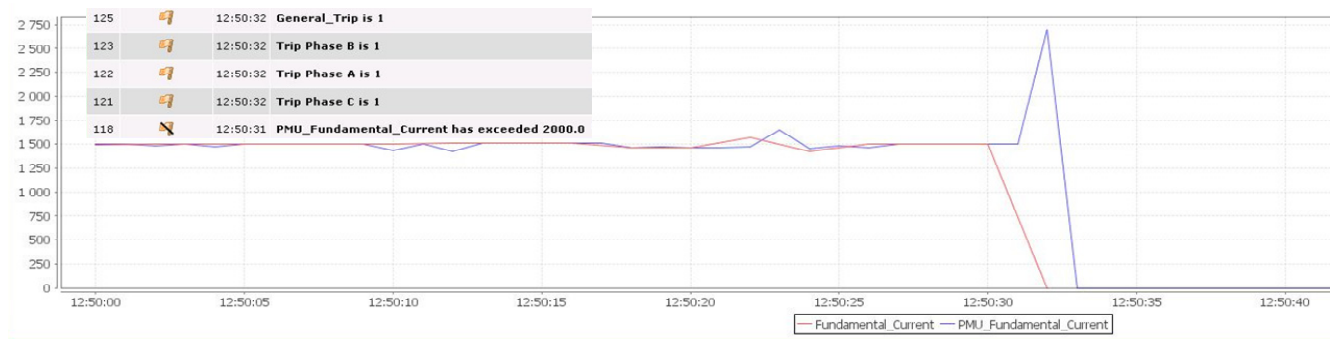

Fig.9: SCADA monitoring of fundamental current measurement received from DNP3 and PMU (IEEE C37.118) protocols

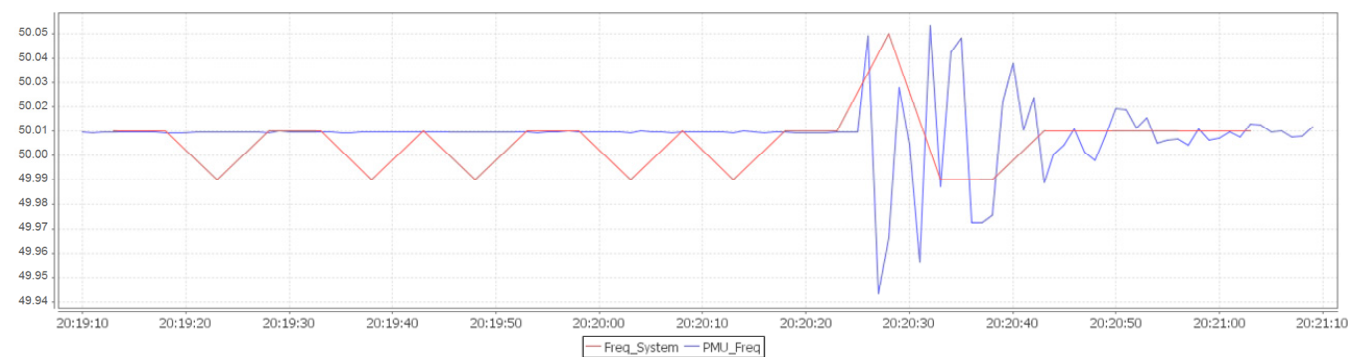

Fig.10: SCADA monitoring of system frequency when mechanical power input of Generator 1 is perturbed with 0.1 pu step.

SCADA BR has the limitation that it takes only two points per second (maximum resolution) when plotting the data. However the database does hold all the data points i.e. all 50 data points received from PMU measurements each second. Figure 10 shows the result of frequency measured by both DNP3 and PMU integrated into SCADA when a step change was applied at the mechanical power input of generator 1 . PMU measurements do update fast, but due to SCADA BR's limitation to update the plot with all the data points, the integration does not provide clear information. The synchrophasor data received by SCADA BR can be plotted as a separate chart using the measurements stored in its data base, as shown in Figure 11. The integration of PMU measurements into SCADA BR is possible however there are limitations.

\section{CONCLUSIONS}

This paper reported the implementation of an open source SCADA using relays in the Smart Transmission System Lab at KTH. The overall implementation of SCADA BR was verified by real-time hardware-in-the-loop simulation of two area four machine power system using Opal-RT's eMEGAsim Real-Time Simulator coupled with protection relays from SEL. PMU measurements are integrated in open source SCADA to make the alarming and visualization system more reliable. The challenges involved in integrating synchrophasor measurements into open source SCADA involve limitations of the update rate of SCADA BR to read the PMU measurements from its database for plotting.

\section{REFERENCES}

[1] J. Marihart, "Communications technology guidelines for EMS / SCADA systems", IEEE Transaction on Power Delivery, Volume 16, Issue 2, April 2001

[2] IEEE Standard for Electric Power System Communications - Distributed Network Protocol DNP3

[3] M.S. Almas et al., "Synhcrophasor Network, Laboratory and Software Applications Developed in the STRONg ${ }^{2}$ rid Project", submitted to IEEE Power and Energy Society, 2014 General Meeting, July 2014.

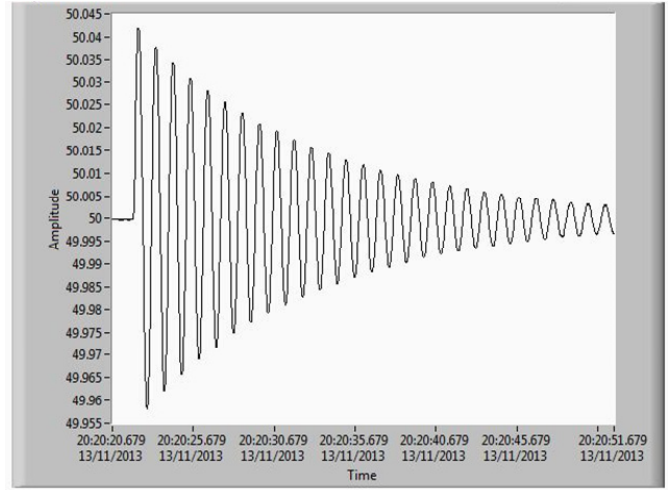

Fig.11: Synchrophasor based measurement plot retrieved from SCADA Database

[4] Opal-RT, "eMEGAsim PowerGrid Real-Time Digital Hardware in the Loop Simulator," available on-line: http:/www.opal-rt.com/.

[5] V. Madani, NASPI Tutorial on IEC 61850 Introductions, NASPI 6185090-5 tutorial workshop Atlanta 2012, Atlanta, GA, USA

[6] "Current and Voltage Amplifiers by Megger," available on-line: http://www.megger.com/cae/story/Index.php?ID=527.

[7] SEL, "SEL-5073 Phasor Data Concentrator (PDC) Software," available on-line: https://www.selinc.com/SEL-5073/

[8] L. Vanfretti, V. H. Aarstrand, M. S. Almas, V. S. Perić and J. O. Gjerde, "A Software Development Toolkit for Real-Time Synchrophasor Applications", IEEE Powertech 2013, Grenoble, France, June 2013

[9] "Open Source- Web Based SCADA-SCADA BR", documentation available online: http://www.scadabr.com.br/

[10] SEL, "Protection Relays by Schweitzer Engineering Laboratories," available on-line: http://www.selinc.com/protection/.

[11] "SEL AcSELerator Quickset - Tool for modifying SEL Relays Settings," Available online: http://www.selinc.com/SEL-5030/

[12] L. Vanfretti, V. H. Aarstrand, M. S. Almas, V. S. Perić and J. O. Gjerde, "A Software Development Toolkit for Real-Time Synchrophasor Applications", IEEE Powertech 2013, Grenoble, France, June 2013

[13] C. Burman \& U. Haque, "Portholes \& plumbing: how AR erases boundaries between 'physical' \& 'virtual' ", Position Paper for W3C Workshop: Augmented Reality on the Web, available online: http://www.w3.org/2010/06/w3car/portholes_and_plumbing.pdf

[14] IoTally, "Pachube Apple Application", available online: https://itunes.apple.com/us/app/iotally/id500127184?mt=8 